University of Nebraska - Lincoln

DigitalCommons@University of Nebraska - Lincoln

$10-29-2004$

\title{
Nanoscale Hydrophobic Interaction and Nanobubble Nucleation
}

T. Koishi

Computational Sciences Division, Advanced Computing Center, RIKEN,Wako, Saitama 351-0198, Japan

S. Yoo

University of Nebraska-Lincoln

K. Yasuoka

Keio University, Yokohama 223-8522, Japan

Xiao Cheng Zeng

University of Nebraska-Lincoln, xzeng1@unl.edu

T. Narumi

High Performance Biocomputing Research Team, Bioinformatics Group, RIKEN Genomic Sciences Center, Yokohama 244-0804, Japan

See next page for additional authors

Follow this and additional works at: https://digitalcommons.unl.edu/chemzeng

Part of the Chemistry Commons

Koishi, T.; Yoo, S.; Yasuoka, K.; Zeng, Xiao Cheng; Narumi, T.; Susukita, R.; Kawai, A.; Furusawa, H.; Suenaga, A.; Okimoto, N.; Futatsugi, N.; and Ebisuzaki, T., "Nanoscale Hydrophobic Interaction and Nanobubble Nucleation" (2004). Xiao Cheng Zeng Publications. 24.

https://digitalcommons.unl.edu/chemzeng/24

This Article is brought to you for free and open access by the Published Research - Department of Chemistry at DigitalCommons@University of Nebraska - Lincoln. It has been accepted for inclusion in Xiao Cheng Zeng Publications by an authorized administrator of DigitalCommons@University of Nebraska - Lincoln. 


\section{Authors}

T. Koishi, S. Yoo, K. Yasuoka, Xiao Cheng Zeng, T. Narumi, R. Susukita, A. Kawai, H. Furusawa, A. Suenaga, N. Okimoto, N. Futatsugi, and T. Ebisuzaki 


\title{
Nanoscale Hydrophobic Interaction and Nanobubble Nucleation
}

\author{
T. Koishi, ${ }^{1}$ S. Yoo, ${ }^{2}$ K. Yasuoka, ${ }^{3}$ X. C. Zeng, ${ }^{2, *}$ T. Narumi,${ }^{4}$ R. Susukita, ${ }^{1}$ A. Kawai, ${ }^{1}$ H. Furusawa, ${ }^{1}$ A. Suenaga, ${ }^{4}$ \\ N. Okimoto, ${ }^{1}$ N. Futatsugi, ${ }^{4}$ and T. Ebisuzaki ${ }^{1}$ \\ ${ }^{1}$ Computational Sciences Division, Advanced Computing Center, RIKEN, Wako, Saitama 351-0198, Japan \\ ${ }^{2}$ Department of Chemistry, University of Nebraska, Lincoln, Nebraska 68588, USA \\ ${ }^{3}$ Department of Mechanical Engineering, Keio University, Yokohama 223-8522, Japan \\ ${ }^{4}$ High Performance Biocomputing Research Team, Bioinformatics Group, RIKEN Genomic Sciences Center, \\ Yokohama 244-0804, Japan
}

(Received 22 July 2004; published 25 October 2004)

\begin{abstract}
We report large-scale atomistic simulation of midrange nanoscale hydrophobic interaction, manifested by the nucleation of nanobubble between nanometer-sized hydrophobes at constrained equilibrium. When the length scale of the hydrophobes is greater than $2 \mathrm{~nm}$, the nanobubble formation shows hysteresis behavior resembling the first-order transition. Calculation of the potential of mean force versus interhydrophobe distance provides a quantitative measure of the strength of the nanoscale hydrophobic interaction.
\end{abstract}

DOI: 10.1103/PhysRevLett.93.185701

Hydrophobic interaction has long been thought of as one of the major forces in stabilizing protein assemblies [1-12]. One of the key characterizations of protein thermal stability is the protein melting temperature $T_{m}$, by which the thermal stability of protein has been measured $[13,14]$, and where the (mesoscopic) protein unfolding reaction bears some resemblance to the macroscopic first-order transition. The classical theory of molecular hydrophobicity [1] assumed that an increased ordering of water occurs around hydrophobic molecular groups, namely, the hydrophobic attraction is mainly driven by the gain in entropy [12]. However, the molecular hydrophobic attraction (e.g., between a pair of methane molecules in water) has been proven short ranged (4-8 $⿱$ ) with the barrier to dissociate comparable to the thermal energy $[4,15,16]$ and is thus unlikely responsible for the thermal stability of protein assemblies [17].

There is also considerable interest in the physics of long-range hydrophobic interaction which has been suggested due to the formation of submicroscopic bubbles on hydrophobic surfaces [1,18-22]. However, a typical range of this interaction is on the order of $10-100 \mathrm{~nm}$ or longer, which is greater than the length scale of small proteins. Recently, Lum, Chandler, and Weeks [9] have developed a theory of hydrophobicity and predicted midrange nanoscopic hydrophobic interaction induced by dewetting (drying) of water near a nanoscale hydrophobe. Berne and co-workers $[23,24]$ have demonstrated the formation of nanobubble (drying) between two model oblate ellipsolids (as large as $1.3 \mathrm{~nm}$ in diameter) via molecular dynamics (MD) simulations. To gain more insight into the midrange nanoscale hydrophobic interaction, we have performed large-scale MD simulations to show that the midrange hydrophobic interaction, manifested by the nucleation of nanobubble between nanometer-sized hydrophobes at constrained equilibrium, is about twice the range of typical molecular hydrophobic interaction (e.g.,
PACS numbers: 64.60.Qb, 64.60.My, 68.03.-g, 82.60.Nh

between methane-methane molecules in water $[15,16])$. Particular attention is placed to the dependence of the interaction on the size of hydrophobes as well as the potential of mean force versus distance curve. When the length scale of the hydrophobes is greater than $2 \mathrm{~nm}$, the nanobubble nucleation (in between the hydrophobes) shows hysteresis behavior, resembling typical macroscopic first-order transition.

Two series of MD simulations aiming at two different aspects of nanoscale hydrophobic interaction were carried out. In the first-series, we performed a constanttemperature and constant-volume MD simulation of nanobubble formation between two hybrid polar/nonpolar nanoassemblies which were held fixed (constrained equilibrium) within a water slab. The water slab is at phase coexistence with the vapor at $T_{R}=298 \mathrm{~K}$ [Fig. 1(a)]. The Nosé-Hoover's method was used to control the temperature. The selection of the vapor-liquid coexistence system is to maintain the ambient condition. The popular SPC/E (extended single point charge) model of water [25] was adopted. Each nanoassembly is composed of $4 \mathrm{~nm}$-sized hydrophobes and a hydrophilic substrate. The OPLS (optimized potentials for liquid simulation) protein model of the $\mathrm{CH}_{3}$ unit [26] was adopted for the nonpolar sites, whereas the polar sites of the hydrophilic substrate were the SPC/E water molecules with fixed center of mass. The long-range charge-charge interaction was computed using the Ewald method.

A special-purpose MD machine, currently the fastest in the world (with peak speed $>70$ Tflops), was employed for the large-scale MD simulation [27]. Initially, $50 \mathrm{ps}$ was used to equilibrate the vapor-liquid system for which all monomer sites on the nanoassemblies were set to be polar. Note that here a MD time step is $1 \mathrm{fs}$. At $50 \mathrm{ps}$, the nanoassemblies were switched instantly to the hybrid nonpolar/polar ones. Another 500 ps was then used to let the system achieve constraint equilibrium. During the 
MD simulation, the distance between the two nanoassemblies $D$ was fixed. When $D=7.1 \AA$, which corresponds to the length scale of one water-layer, four nanobubbles (or cavities) nucleate and grow spontaneously in between the four pairs of hydrophobes [the left snapshot in Fig. 1(b)]. When $D=10.2 \AA$, which corresponds to the length scale of two water layers, the spontaneous nanobubble formation [the middle snapshot in Fig. 1(b)] occurred only in between the larger hydrophobes $(5 \times 5$ and $6 \times 6)$. This result clearly demonstrates that the extent to which the hydrophobes interact depends sensitively on the size of the hydrophobes. As $D$ was further enlarged to $13.4 \AA$ the spontaneous nanobubble formation was never observed, at least within the time (500 ps) of the MD simulation. However, when we used a different initial water configuration in the case of $D=13.4 \AA$, that is to let a cavity preexist in between the two nanoassemblies, we found that the cavity region between the smaller hydrophobes $(3 \times 3$ and $4 \times 4)$ was filled up with water in less than $100 \mathrm{ps}$, whereas the cavity (nanobubble) was sustained in between the two larger hydrophobes $(5 \times 5$ and $6 \times 6)$ during ensuing $500 \mathrm{ps}$ MD run [the right snapshot in Fig. 1(b)]. An additional 500 ps MD run was also performed and it was found that the two nanobubbles were still stable. This dependence of the state of nanobubble on the initial configuration of system indicates that the formation of the nanobubbles bears some resemblance to the first-order capillary evaporation [24,28-30]. As such, the nanoscopic "phase" change can be initiated by a nucleation process, and the growth of the "nanobubble phase" occurs only after the fluctuation achieves a critical size. Here, the mesoscopic nucleation is heterogeneous due to the weakly dewetting of the proximal water to the hydrophobes $[9,24]$. Unlike the homogeneous bubble nucleation, here the nanobubbles cannot grow indefinitely but are limited by the size of hydrophobes. The larger the hydrophobes, the greater the stabilized nanobubbles. Finally, when the distance $D$ is enlarged beyond the length scale of four water layers $(16.0 \AA)$, stable nanobubbles cannot be observed. This implies that the hydro-
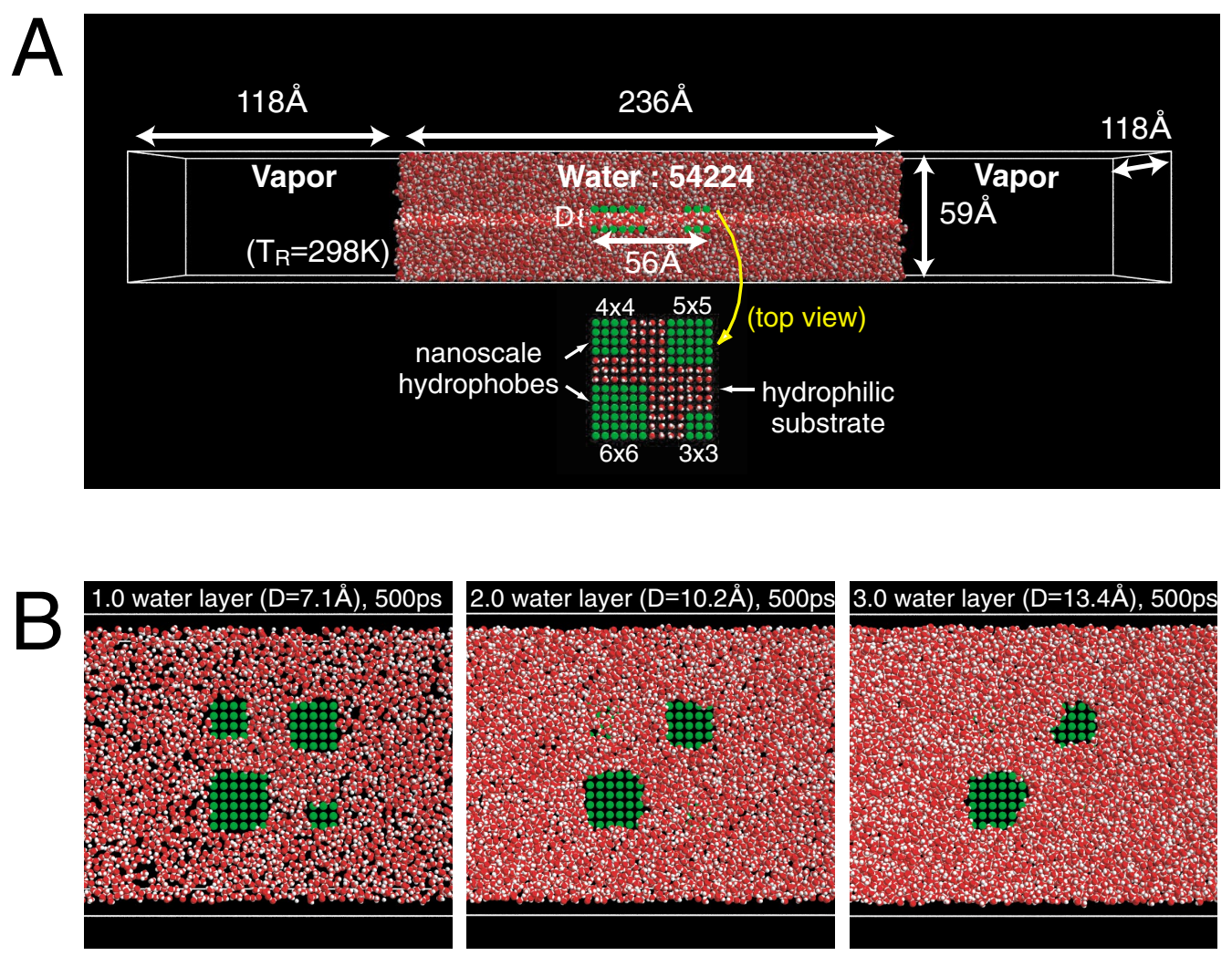

FIG. 1 (color). (a) The simulation cell contains a slab of SPC/E water (coexisting with the vapor at $T_{R}=298 \mathrm{~K}$ ) and two identical hybrid polar/nonpolar nanoassemblies, each containing four nanoscale hydrophobes. The nanoassembly has totally $13 \times 13=169$ monomer sites, among them 83 polar sites were SPC/E water molecules (the center of mass of water molecule was fixed but the molecule was allowed to rotate). The four nanoscale hydrophobes have totally 86 nonpolar sites, and each site was modeled as the $\mathrm{OPLS} \mathrm{CH}_{3}$ unit. The length scale of the square-shaped hydrophobes ranges from $1.269 \mathrm{~nm}$ (containing $3 \times 3=9$ nonpolar sites) to $2.585 \mathrm{~nm}$ (containing $6 \times 6=36$ nonpolar sites). The water-vapor interfaces were set normal to the $z$ axis and the two planar nanoassemblies immersed in the water slab were normal to the $x$ axis. Periodic boundary conditions were applied in all directions. (b) Top views of the snapshot of the highlighted water-layer sandwiched in between the two nanoassemblies shown in (a). To show the snapshots, the upper nanoassembly is removed. The distance $D$ between the two nanoassemblies is 7.1, 10.2, and $13.4 \AA$, which corresponds to the length scale of one, two, and three layers of water, respectively. 
phobic interaction becomes very tenuous beyond the range of $16.0 \AA$, at least between the largest $6 \times 6$ hydrophobes considered here. To achieve longer ranges of hydrophobic interaction, larger sizes of hydrophobes are required [12].

To gain more quantitative understanding about the strength of the nanoscale hydrophobic interaction, we performed another series of MD simulations to calculate the potential of mean force (PMF) as a function of the interhydrophobe distance $D$. Note that the slope of the PMF- $D$ curve characterizes the magnitude of the hydrophobic force (effective attraction) between the hydrophobes. The maximum PMF can be also viewed as the work required to disjoin the two hydrophobes (when initially in contact) in water. The system considered was similar to that for the hybrid polar/nonpolar nanoassemblies [Fig. 1(a)], which consists of 5560 SPC/E water molecules and two purely $6 \times 6$ hydrophobes. The entire vapor-liquid system has the dimensions of $65.0 \AA \times$ $52.0 \AA \times 80.0 \AA$. The Gibbs free-energy changes $\Delta G / k_{B} T$ vs the interhydrophobe distance $D$ (defined as the distance from the center of mass of one hydrophobe to the opposing one) were evaluated by means of the umbrella-sampling method $[31,32]$. To this end, a biasing potential $\quad U_{b}(D)=k_{l}\left(D_{l}-D\right)^{n_{l}} H\left(D_{l}-D\right)+k_{u}(D-$ $\left.D_{u}\right)^{n_{u}} H\left(D-D_{u}\right)+k D$ was used, where $H(x)$ is 0 for $x<$
0 and 1 for $x>0$. The biasing potential $U_{b}(D)$ constrains the interhydrophobe distance within the window $D_{l} \leq$ $D \leq D_{u}$. Each window has the separation of $D_{u}-D_{l}=$ $0.5 \AA$. We set $k_{l}=k_{u}=500 \mathrm{kcal} / \mathrm{mol}$ and $n_{l}=n_{u}=3$. The last term of $U_{b}(D)$ is used to achieve a more uniform probability distribution within a single window. The probability distribution, $p(D)$, as a function of $D$, is related to the PMF via the equation $\Delta G / k_{B} T=$ $-\ln \left[p(D) / p\left(D_{0}\right)\right]$ where $D_{0}$ is a reference distance $\left(D_{0}=\right.$ $4.05 \AA$ ). During the MD simulation, the system was equilibrated for 100 ps followed by another 500 ps production run in which data was accumulated in every ten MD step.

The calculated PMF curves, as described by the freeenergy change from the reference $\left(D_{0}\right)$, are plotted in Fig. 2 for two given temperatures $(T=298.15 \mathrm{~K}$ and $\left.T^{\prime}=348.15 \mathrm{~K}\right)$. In Fig. 2 , snapshots of a portion of the system in the expansion process, as highlighted by the circles $E_{1} / E_{2} / E_{3} / E_{4}$, are also displayed, as well as those in the backward contraction process, highlighted by $\mathrm{C}_{1} / \mathrm{C}_{2}$. First, as shown in the first-series MD simulation, for $D<10.5 \AA$ the calculated PMF is independent of the history (expansion or contraction), whereas for $D>10.5$ it does depend on the process. The onset of the latter behavior bears resemblance to the hysteresis behavior in typical first-order phase transition. Second, it can be seen
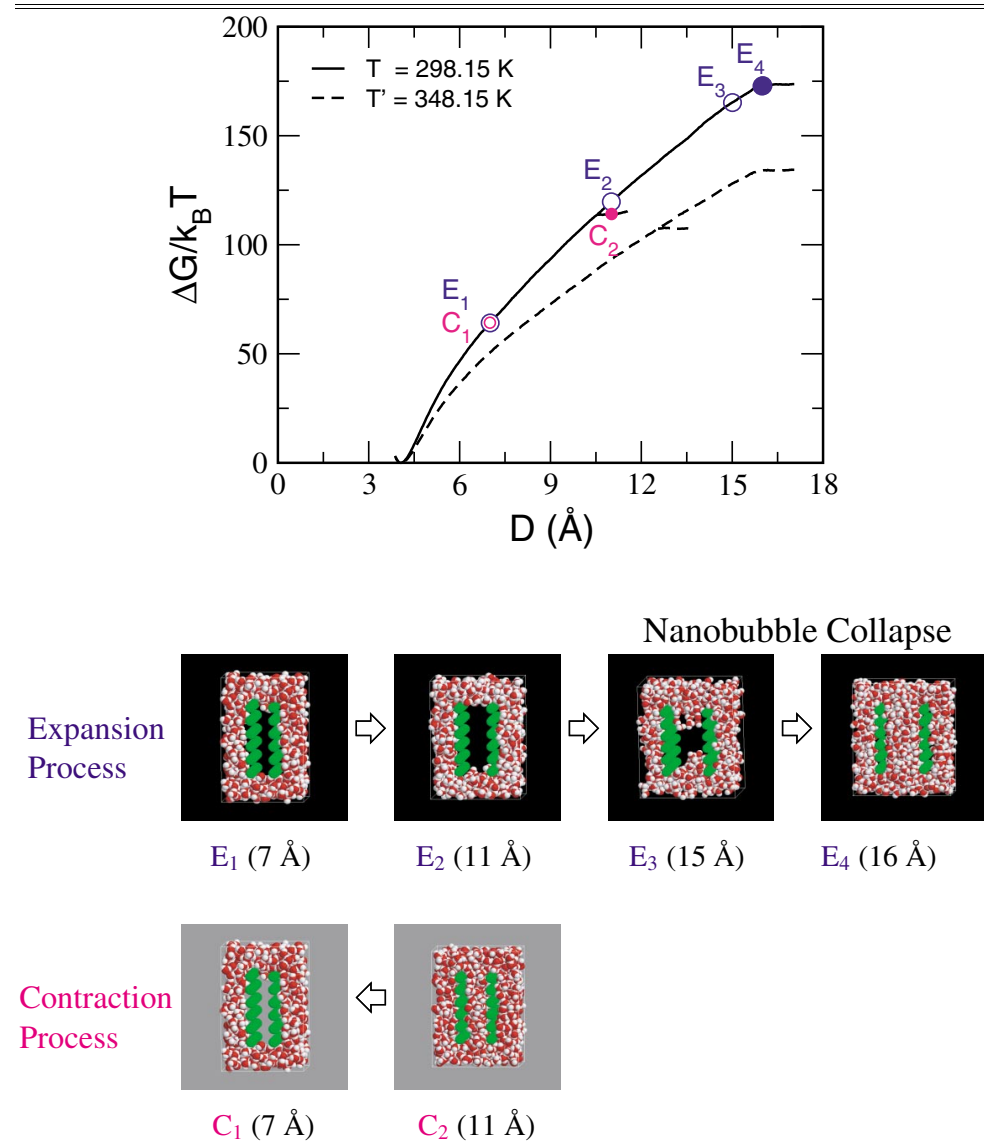

FIG. 2 (color). The calculated PMF curve $\left(\Delta G / k_{B} T\right.$ vs the interhydrophobe distance $D$ ) for two $6 \times 6$ hydrophobes in the expansion and the contraction process. The open circles highlight the stable nanobubble state, whereas the solid circles highlight the nanobubblecollapsing state. Snapshots corresponding to these specific states are displayed. 
from Fig. 2 that to form a one water-layer size nanobubble, the work of formation amounts to about $65 k_{B} T$ (where $k_{B}$ is the Boltzmann constant). As a comparison, the barrier to dissociation for a methane pair (corresponding to intermolecular distance about $6 \AA$ ) in water is on the order of $1 k_{B} T[4,15,16]$. Moreover, the midrange hydrophobic interaction is more than twice longer in range than that of molecular hydrophobic interaction between the methane pair. Third, as the temperature is increased by $50 \mathrm{~K}$, the PMF is reduced appreciably, e.g., from $65 k_{B} T$ to about $50 k_{B} T$ at $D \sim 7$. As a consequence, the hydrophobic force on the hydrophobes (measured by the negative gradient of the PMF) is also reduced by increasing the temperature. In contrast, it has been shown that the molecular hydrophobic interaction between two methane molecules becomes slightly stronger with increasing the temperature [16].

The PMF curves of nanoscale hydrophobic interaction might offer some new insights into the thermal stability of protein (e.g., the hyperthermal proteins). Why is a folded (or even misfolded) protein stable against mild temperature increase above the room temperature $T_{R}$ ? We show in Fig. 2 that when the nanobubble collapses the PMF levels off. This is a consequence of the simple rigidnanoassembly model considered here. If the conformational degrees of freedom were allowed as in real proteins, the conformational entropy would increase during the expansion (unfolding). The total free-energy change (or the PMF) will be expected to decrease at some turning point and as a result, the free-energy curves will show a maximum (the free-energy barrier). If the maximum is viewed as a transition state of the "unfolding reaction," and the mean interhydrophobic-patch distance within the "hydrophobic core" is viewed as the unfolding reaction coordinate, then the free-energy change along the unfolding pathway will describe the work required to disjoin the hydrophobic core. In this simple picture, the unfolding transition state corresponds to the state of the nanobubble collapsing at which the hydrophobic interaction within the hydrophobic core is disrupted. Moreover, our calculation indicates that when the temperature is modestly increased above $T_{R}$ (with other conditions fixed), the freeenergy barrier will be reduced, but still significantly higher than typical thermal energy $\left(\sim k_{B} T\right)$. Hence, although reduced, the still high free-energy barrier may have implication toward understanding the net stability of protein assembles against modest temperature increase, as well as the enhancement of kinetic unfolding rate constant with increasing temperature [33,34].

This research is supported by grants from NSF (ITR and NIRT), DOE's Office of Basic Energy Sciences (Division of Materials Sciences and Engineering), and Nebraska Research Initiative, by the Japan Ministry of Education, and by the Research Computing Facility at UNL. T. K. and N.O. are supported by the special research program in RIKEN. X. C. Z. is grateful for valu- able discussions with Professor David Chandler, Professor Joan-Emma Shea, and Professor Xueyu Song.

*Corresponding author.

Email address: xczeng@phase1.unl.edu

[1] H.S. Frank and M.W. Evans, J. Chem. Phys. 13, 507 (1945).

[2] W. Kauzmann, Adv. Protein Chem. 14, 1 (1959).

[3] F. H. Stillinger, J. Solution Chem. 2, 141 (1973).

[4] L. R. Pratt and D. Chandler, J. Chem. Phys. 67, 3683 (1977).

[5] K. A. Dill, Biochemistry 29, 7133 (1990).

[6] W. Blokzijl and J. B. F. N. Engberts, Angew. Chem. Int. Ed. Engl. 32, 1545 (1993).

[7] S. Garde, G. Hummer, and M. Paulaitis, Faraday Discuss. 103, 125 (1996).

[8] Y.-K. Cheng and P. J. Rossky, Nature (London) 392, 696 (1998).

[9] K. Lum, D. Chandler, and J. D. Weeks, J. Phys. Chem. B 103, 4570 (1999).

[10] J. M. Sorenson et al., J. Phys. Chem. B 103, 5413 (1999).

[11] D. M. Huang and D. Chandler, Proc. Natl. Acad. Sci. U.S.A. 97, 8324 (2000).

[12] P. Ball, Nature (London) 423, 25 (2003).

[13] T. E. Creighton, Proteins (Freeman, New York, 1991).

[14] W. J. Becktel and J. A. Schellman, Biopolymers 26, 1859 (1987).

[15] D. E. Smith, L. Zhang, and A. D. J. Haymet, J. Am. Chem. Soc. 114, 5875 (1992)

[16] S. Lödemann, H. Schreiber, R. Abseher, and O. Steinhauser, J. Chem. Phys. 104, 286 (1996).

[17] P. R. ten Wolde, J. Phys. Condens. Matter 14, 9445 (2002).

[18] P. Atard, Adv. Colloid Interface Sci. 104, 75 (2003).

[19] X. Zhang, Y. Zhu, and S. Granick, J. Am. Chem. Soc. 123, 6736 (2001).

[20] D. Schwendel et al., Langmuir 19, 2284 (2003).

[21] T. R. Jensen et al., Phys. Rev. Lett. 90, 086101 (2003).

[22] R. Steitz et al., Langmuir 19, 2409 (2003).

[23] A. Wallquist and B. J. Berne, J. Phys. Chem. 99, 2893 (1995).

[24] X. Huang, C. J. Margulis, and B. J. Berne, Proc. Natl. Acad. Sci. U.S.A. 100, 11953 (2003).

[25] H. J. C. Berendsen, J. R. Grigera, and T. P. Straatsma, J. Phys. Chem. 91, 6269 (1987).

[26] W. L. Jorgensen and J. Tirado-Rives, J. Am. Chem. Soc. 110, 1657 (1988).

[27] Details of the MD machine are given in T. Narumi et al., Proceedings of Supercomputing 2000.

[28] D. Chandler, Nature (London) 417, 491 (2002).

[29] T. M. Truskett, P. G. Debenedetti, and S. Torquato, J. Chem. Phys. 114, 2401 (2001).

[30] D. Bratko, R. A. Curtis, H.W. Blanch, and J. M. Prausnitz, J. Chem. Phys. 115, 3873 (2001).

[31] D. Chandler, Introduction to Modern Statistical Mechanics (Oxford University, New York, 1987).

[32] S. Yoo, Y. A. Lei, and X. C. Zeng, J. Chem. Phys. 119, 6083 (2003).

[33] U. Mayor et al., Nature (London) 421, 863 (2003).

[34] W. Y. Yang and M. Gruebele, Nature (London) 423, 193 (2003). 